\title{
On the one-dimensional reggeon model: eigenvalues of the Hamiltonian and the propagator
}

\author{
M. A. Braun ${ }^{1}$, E. M. Kuzminskii ${ }^{1}$, A. V. Kozhedub ${ }^{2}$, A. M. Puchkov ${ }^{1}$, M. I. Vyazovsky ${ }^{1, a}$ \\ ${ }^{1}$ Department of High Energy physics, Saint-Petersburg State University, 198504 Saint Petersburg, Russia \\ ${ }^{2}$ Department of Computational Physics, Saint-Petersburg State University, 198504 Saint Petersburg, Russia
}

Received: 15 July 2019 / Accepted: 31 July 2019 / Published online: 8 August 2019

(C) The Author(s) 2019

\begin{abstract}
The effective reggeon field theory in zero transverse dimension ("the toy model") is studied. The transcendental equation for the eigenvalues of the Hamiltonian of this theory is derived and solved numerically. The found eigenvalues are used for the calculation of the pomeron propagator.
\end{abstract}

\section{The one-dimensional quantum reggeon model}

The effective theory of reggeon interaction (the Gribov model) was introduced in [1] as a set of diagrammatic rules with an infinite number of vertices. In $[2,3]$ by means of the renormalization group method it was shown that at high energies only the three-reggeon interactions are important. In [2] the diagrammatic rules were reconsidered as Feynman rules for the Euclidean field theory in three dimensions with the Lagrangian density

$$
\begin{aligned}
\mathcal{L}= & \frac{1}{2}\left(\Phi^{+} \partial_{y} \Phi-\Phi \partial_{y} \Phi^{+}\right)-\mu \Phi^{+} \Phi-\alpha^{\prime} \vec{\nabla} \Phi^{+} \cdot \vec{\nabla} \Phi \\
& +i \lambda \Phi^{+}\left(\Phi+\Phi^{+}\right) \Phi .
\end{aligned}
$$

Here $\Phi(y, \vec{b})$ is for the complex reggeon (pomeron) field, $\Phi^{+}(y, \vec{b})$ is for the conjugated field, $y$ is for the rapidity parameter (logarithm of total energy), and $\vec{b}$ is for the twodimensional impact parameter. The parameters $\mu=\alpha(0)-1$ and $\alpha^{\prime}=\alpha^{\prime}(0)$ are defined by the pomeron Regge trajectory $j=\alpha(t)$. The effective coupling constant $\lambda$, because of physical reasons, has to be real and positive. One has also to add field sources for $\Phi, \Phi^{+}$which describe the interaction of reggeons with scattering particles-hadrons. It is worth to note that the theory of Eq. (1) arises as a continuous limit in the two-dimensional directed bond percolation model [4].

Electronic supplementary material The online version of this article (https://doi.org/10.1140/epjc/s10052-019-7187-4) contains supplementary material, which is available to authorized users.

a e-mail: ovsky@MV8660.spb.edu
At the same time, in a number of references [5-9] a simplified one-dimensional model was considered,

$$
\begin{aligned}
\mathcal{L}= & \frac{1}{2}\left(\Phi^{+} \partial_{y} \Phi-\Phi \partial_{y} \Phi^{+}\right) \\
& -\mu \Phi^{+} \Phi+i \lambda \Phi^{+}\left(\Phi+\Phi^{+}\right) \Phi,
\end{aligned}
$$

where $\Phi, \Phi^{+}$depend only on $y$. The one-dimensional theory may be thought of as the lowest order approximation of the three-dimensional theory with $\alpha^{\prime} \rightarrow 0$, since in this case the dynamic connection between fields with different impact parameters $\vec{b}$ is lost. To a certain extent it can correspond to physical reality, since the slope of the pomeron trajectory $\alpha^{\prime} \sim 0.2 \mathrm{GeV}^{-2}$ may be considered small in comparison to the momenta scale in hadron scattering processes. However, the one-dimensional model also serves as a "toy model" which allows one to study the correlation between the results given by the perturbative approach and the non-perturbative methods. Conclusions drawn from the analysis of this model can be significant for studying more complicated and realistic theories, including quantum chromodynamics. It is the one-dimensional model that is considered in the presented work.

The theory of Eq. (2) describes a first-order formalism. Besides, this is an Euclidean field theory in the sense that the rapidity $y$ may be understood as the imaginary time. The canonical quantization in imaginary time leads to the commutation relation

$\left[\Phi, \Phi^{+}\right]=1$,

which allows one to interpret $\Phi^{+}$as the creation operator and $\Phi$ as the annihilation operator. The Hamiltonian has the form [9]

$H=-\mu \Phi^{+} \Phi+i \lambda\left(\Phi^{+} \Phi^{+} \Phi+i \lambda \Phi^{+} \Phi \Phi\right)$.

Following [9], we use a normal order of operators. For the Hamiltonian (4) the existence of the Fock space is postulated. Quantum field theory (2) without spatial dimensions is 
equivalent to one-dimensional quantum mechanics, at least in the perturbation (in the coupling constant) theory framework. It is important that the perturbation theory works well for $\mu<0$, when eigenvalues of the unperturbed Hamiltonian are bounded from below.

In Ref. [10] this theory was considered in the "loopless" approximation, when one of the terms inside brackets in (4) is ignored. In this case the theory becomes integrable. However, in $[11,12]$ the evolution in rapidity of the hadron scattering amplitude in the framework of the one-dimensional reggeon model was studied numerically and it was shown that the difference between the full theory and the "loopless" approximation becomes substantial at high $\lambda$. Thus it is meaningful to develop methods that are not connected with the perturbation theory in $\lambda$.

In [13] the eigenvalues of the Hamiltonian (4) were found for values of $\mu / \lambda=1,3,5$ and the propagator at $\mu / \lambda=5$ was calculated, although neither the calculational procedure nor the precision were reported. In this paper we concentrate on the theoretical problems related to the Hamiltonian in the complex plane and on the method of calculation of its eigenvalues and eigenfunctions. We enlarge the domain of the values of $\mu / \lambda$ to include negative ones for comparison with the perturbative approach.

\section{Eigenvalues problem}

In this section we mostly reproduce the results of [9], which serve as a starting point of our study. It is convenient to use the Bargmann representation for the Fock space in the form of the Hilbert space of analytical functions, in order to use methods from the differential equations theory. This representation is introduced by the relation

$\psi(z)=\left\langle 0\left|\mathrm{e}^{\Phi z}\right| \psi\right\rangle$

for a wave function. By construction $\psi(z)$ is an analytical function in $z$. In the Bargmann representation multiplication and differentiation correspond to the creation and annihilation operators,

$\Phi^{+} \rightarrow z, \quad \Phi \rightarrow \frac{d}{d z}$,

the basis Fock state $|n\rangle=\left(\Phi^{+}\right)^{n}|0\rangle$ then corresponds to $\psi(z)=z^{n}$. The standard scalar product of the Fock space in the frame of analytical functions $\psi(z)$ is reproduced by the formula

$\left\langle\psi_{1} \mid \psi_{2}\right\rangle=\int \frac{d z d z^{*}}{2 \pi i} \mathrm{e}^{-z z^{*}} \psi_{1}^{*}(z) \psi_{2}(z)$.

Different basis states are orthogonal, which is easily seen for $\psi_{2}(z)=z^{n}$ and $\psi_{1}^{*}(z)=\left(z^{*}\right)^{m}$, if one carries out in (3) the integration over $\arg (z)$. The basis state norm is defined by the integral over absolute value of $z$ (here $t=|z|^{2}$ ):

$$
\begin{aligned}
<n \mid n> & =\int_{0}^{\infty} d|z| \cdot 2|z| \mathrm{e}^{-|z|^{2}}|z|^{2 n} \\
& =\int_{0}^{\infty} d t \mathrm{e}^{-t} t^{n}=\Gamma(n+1)=n ! .
\end{aligned}
$$

The Hamiltonian (4) in the Fock-Bargmann representation takes the form

$H=-\mu z \frac{d}{d z}+i \lambda z \frac{d^{2}}{d z^{2}}+i \lambda z^{2} \frac{d}{d z}$.

We want to find the eigenvalues and eigenstates of the Hamiltonian. The equation

$H \psi=E \psi$

is a linear differential equation of the second order (of the Heun class; see the next section) the general solution of which is a linear combination of two linearly independent functions. Their asymptotical behavior at $z=0$ is found to be

$\psi_{1}(z) \underset{z \rightarrow 0}{\sim} z$ or $\psi_{2}(z) \underset{z \rightarrow 0}{\sim} c_{1}+c_{2} z \ln z$

Because of analyticity of $\psi(z)$, one has to have $c_{2}=0$, which is possible only when $E=0$. In this case the normalizable solution in Fock-Bargmann space $\psi_{0}(z)=1$ is the vacuum wave function, which has no physical meaning for reggeon theory (from this point of view the state $|0\rangle$ describes the absence of interaction of physical particles with the reggeon). Therefore, the second asymptotics is to be excluded.

The asymptotics in the vicinity of infinity is also known,

$\psi_{3}(z) \underset{z \rightarrow \infty}{\sim}$ const, $\quad \psi_{4}(z) \underset{z \rightarrow \infty}{\sim} \frac{1}{z} \mathrm{e}^{-\frac{1}{2} z^{2}-\frac{i \mu}{\lambda} z}$.

It is worth to note that the asymptotical behavior (12) depends on the direction in the complex plane.

Let us begin with the case $\mu<0$, which corresponds to the domain of the applicability of the perturbation theory. As in [9] we impose the condition of a finite Bargmann norm. Since the asymptotics of the integrand in (7) for $\left\langle\psi_{4} \mid \psi_{4}\right\rangle$ is

$\mathrm{e}^{-\left|z^{2}\right|}\left|\frac{1}{z} \mathrm{e}^{-\frac{z^{2}}{2}-\frac{i \mu}{\lambda} z}\right|^{2} \simeq \frac{1}{|z|^{2}} \mathrm{e}^{\frac{2 \mu}{\lambda} \operatorname{Im} z} \mathrm{e}^{-2(\operatorname{Re} z)^{2}}$,

the integral (7) cannot converge on $\operatorname{Im} z$ if a wave function has asymptotics $\psi_{4}$ on the negative imaginary axis $z$ (it is always assumed that $\lambda>0$ ). A solution with asymptotics $\psi_{3}$ in this direction will have a finite norm. Thus the quantization condition is $\psi \sim \psi_{3}$, when $z \rightarrow-i \infty$.

For further convenience the following substitution will be used:

$z=-i \sqrt{2} x$ 
The eigenfunction equation $H \psi=E \psi$ may be rewritten (for $\lambda \neq 0$ ) in the form

$x \psi^{\prime \prime}+\left(\frac{\mu \sqrt{2}}{\lambda} x-2 x^{2}\right) \psi^{\prime}+\frac{E \sqrt{2}}{\lambda} \psi=0$,

where a prime throughout denotes a derivative with respect to $x$. Asymptotical boundary conditions for the solution $\psi(x)$, analytical in the entire complex plane $x$, have to be set on the ray $x \in[0,+\infty)$ :

$\psi \underset{x \rightarrow 0}{\sim} x, \quad \psi \underset{x \rightarrow+\infty}{\sim}$ const.

Note that in the case $\mu>0$, the finite Bargmann norm condition $\psi \sim \psi_{3}$ has to be put on the positive imaginary axis $z$, i.e. on the ray $x \in(-\infty, 0]$. After substitution $x \rightarrow-x$ the equation takes the form

$x \psi^{\prime \prime}+\left(\frac{-\mu \sqrt{2}}{\lambda} x-2 x^{2}\right) \psi^{\prime}+\frac{-E \sqrt{2}}{\lambda} \psi=0$

with the same boundary conditions (16). It is equivalent to the solution of Eq. (15) with $\mu \rightarrow-\mu<0, E \rightarrow-E$. Calculations made in Sect. 5 show that for $\mu<0$ all values $E$ are positive. Therefore the solutions of the Eq. (17) correspond to unphysical negative values of $E$. This leads, e.g., to the infinite growth of the propagator (see Sect. 6) when $y \rightarrow+\infty$. Thus such a formulation of the problem seems to be incorrect in the case $\mu>0$. In Sect. 5 for $\mu>0$ the same conditions (16) for Eq. (15) on eigenfunctions were used. As a result, all found eigenvalues $E$ are positive.

When $\mu=0$, the finiteness of the norm does not exclude both asymptotics $\psi \sim \psi_{3,4}$ on the ray $x \in[0,+\infty)$ and on the ray $x \in(-\infty, 0]$. In this case the conditions (16) can be used as well, excluding the asymptotics $\psi_{4}$, and all the eigenvalues are found to be positive. Furthermore, in Sect. 6 the completeness property of the eigenfunctions found was partially checked, and as a result the necessary solutions were not lost.

In [9] it was proven that the spectral representation of the $S$-matrix of the theory (4) exists and it is analytical in $\mu$ on the entire real axis $\mu$. The choice of the quantization conditions (16) for all values of $\mu$ is to be understood in the sense of such an analytical continuation. The positiveness of the eigenvalues found indicates the correctness of this approach.

The Hamiltonian (9) is non-Hermitian with respect to the standard scalar product (7). Hence the question arises, whether the eigenfunctions defined by the finite norm condition form the complete basis. The answer is given by the transformation procedure of (9) to the Hermitian form, proposed in [7]. On the negative imaginary axis $z=-i q$, $q=\sqrt{2} x>0$, and

$H \equiv \lambda q\left(-\frac{\mathrm{d}^{2}}{\mathrm{~d} q^{2}}+\left(q-\frac{\mu}{\lambda}\right) \frac{\mathrm{d}}{\mathrm{d} q}\right)$.
By means of a similarity transformation $\tilde{H}=\mathrm{e}^{-F(q)} H \mathrm{e}^{F(q)}$ with $F(q)=\mathrm{e}^{\frac{1}{4}\left(q-\frac{\mu}{\lambda}\right)^{2}}$ the term with a first-order derivative may be annihilated. After substitution $q=\xi^{2}, \xi>0$ and one more transformation we have

$$
\begin{aligned}
H_{\xi} & =\xi^{-\frac{1}{2}} \tilde{H} \xi^{\frac{1}{2}} \\
& =\frac{\lambda}{4}\left(-\frac{\mathrm{d}^{2}}{\mathrm{~d} \xi^{2}}+\frac{3}{4 \xi^{2}}+\xi^{2}\left(\left(\xi^{2}-\frac{\mu}{\lambda}\right)^{2}-2\right)\right),
\end{aligned}
$$

taking the form of a Hermitian Hamiltonian with a singular potential. The similarity transformation from $H$ to $H_{\xi}$ is non-unitary, but bijective. The equation $H \psi=E \psi$ is then equivalent to $H_{\xi} \varphi=E \varphi$, where

$\varphi(\xi)=\xi^{-\frac{1}{2}} \mathrm{e}^{-\frac{1}{4}\left(\xi^{2}-\frac{\mu}{\lambda}\right)^{2}} \psi\left(z=-i \xi^{2}\right)$

is an analytical function in $\xi$ in the vicinity of the half-axis $(0,+\infty)$ and it is finite when $\xi \rightarrow 0$. The choice of functions $\psi(z) \underset{z \rightarrow 0}{\sim} z$ means that $\varphi(\xi) \underset{\xi \rightarrow 0}{\sim} \xi^{3 / 2}$-this behavior corresponds to the angular momentum barrier for the potential in (19).

The asymptotics of eigenfunctions at $\xi \rightarrow+\infty$, corresponding to (12), is defined by the relations

$\varphi_{3}(\xi) \sim \xi^{-\frac{1}{2}} \mathrm{e}^{-\frac{1}{4}\left(\xi^{2}-\frac{\mu}{\lambda}\right)^{2}}, \quad \varphi_{4}(\xi) \sim \xi^{-\frac{5}{2}} \mathrm{e}^{\frac{1}{4} \xi^{4}-\frac{\mu}{2 \lambda} \xi^{2}}$.

The finiteness of the norm of $\varphi(\xi)$ in the space $L_{2}([0,+\infty))$ excludes the second asymptotics, which is equivalent to the choice of asymptotics $\psi_{3}$ for $\psi(z)$, i.e. the Bargmann norm finiteness. Thus the previously formulated problem of finding the spectrum of the Hamiltonian $H$ is equivalent to the problem of finding the spectrum of the Hermitian operator $H_{\xi}$ in the space $L_{2}([0,+\infty))$. The eigenfunctions of this problem form a complete basis; all the eigenvalues, common for $H$ and $H_{\xi}$, are real. Note that the Hermiticity of $H_{\xi}$ does not depend on the sign of $\mu$.

\section{Biconfluent Heun equation}

The canonical form of the biconfluent Heun equation is ( $\alpha, \beta, \gamma, \delta$ are constant parameters)

$$
\begin{aligned}
& x \psi^{\prime \prime}(x)+\left(1+\alpha-\beta x-2 x^{2}\right) \psi^{\prime}(x) \\
& +\left((\gamma-\alpha-2) x-\frac{1}{2}(\delta+(1+\alpha) \beta)\right) \psi(x)=0 .
\end{aligned}
$$

By means of the transformation

$u(x)=x^{\frac{1+\alpha}{2}} \exp \left(-\frac{\beta}{2} x-\frac{1}{2} x^{2}\right) \psi(x)$,

Equation (22) is brought into the so-called normal form, 


$$
\begin{aligned}
& u^{\prime \prime}(x)+\left(\frac{1}{4}\left(1-\alpha^{2}\right) \frac{1}{x^{2}}-\frac{1}{2} \delta \frac{1}{x}\right. \\
& \left.+\gamma-\left(\frac{\beta}{2}\right)^{2}-\beta x-x^{2}\right) u(x)=0 .
\end{aligned}
$$

Equation (15), rewritten as

$x \psi^{\prime \prime}(x)+\left(-\beta x-2 x^{2}\right) \psi^{\prime}(x)-\frac{\delta}{2} \psi(x)=0$,

is the canonical form of the biconfluent Heun equation with the parameters

$\alpha=-1, \quad \beta=-\frac{\sqrt{2} \mu}{\lambda}, \quad \gamma=1, \quad \delta=-\frac{2 \sqrt{2} E}{\lambda}$.

The behavior of the solutions of Eq. (22) is well known [14]. Let us recall some main facts about it (useful formulas may also be found in [15]). The equation has two singular points, a regular one at $x=0$ and an irregular one at $x=\infty$ with the rank of singularity $R=3$. A solution of the equation cannot have any finite singularities apart from $x=0$.

Generally, the leading asymptotics near zero is a power $x^{\frac{\alpha \pm 1}{2}}$. Our case $\alpha=-1$ is special in the sense that only one of the solutions can be represented in the form of a power series (Frobenius series)

$\psi(x)=\sum_{n=0}^{\infty} c_{n} x^{n+1}$

converging in the entire complex plane. Its coefficients are defined (up to a common factor) by the three-term recurrence relation

$n(n+1) c_{n}=\left(n \beta+\frac{\delta}{2}\right) c_{n-1}+2(n-1) c_{n-2}, \quad c_{0}=1$.

We will always consider $c_{0}=1$ for solutions. Thus $\psi(x) \sim x$ when $x \rightarrow 0$. The second linearly independent solution with $\alpha=-1$ has the form

$\tilde{\psi}(x)=\psi(x) \ln x+f(x)$,

where $f(x)$ is analytical function in $x$; it has to be excluded, because it has a logarithmic branch point $x=0$. Only the solutions of the type (27), analytical in the entire complex plane $x$, have to be considered.

In the vicinity of $x=\infty$ one can choose as two linearly independent solutions of Eq. (25) the functions $\psi_{3}$ and $\psi_{4}$, defined by the asymptotic power series (Thomè series)

$$
\begin{aligned}
& \psi_{3}(x) \underset{x \rightarrow \infty}{\sim} \sum_{n=0}^{\infty} a_{n}^{3} x^{-n}, \\
& \psi_{4}(x) \underset{x \rightarrow \infty}{\sim} \frac{1}{x} \exp \left(x^{2}+\beta x\right) \sum_{n=0}^{\infty} a_{n}^{4} x^{-n} .
\end{aligned}
$$

As can be seen, the asymptotics (12) are the first terms of the regular expansions (30). The coefficients of these expansions are fully defined by the recurrence relations

$$
\begin{aligned}
& 2 n a_{n}^{3}=\left(-(n-1) \beta+\frac{\delta}{2}\right) a_{n-1}^{3}-(n-1)(n-2) a_{n-2}^{3}, \\
& 2 n a_{n}^{4}=-\left(n \beta+\frac{\delta}{2}\right) a_{n-1}^{4}+n(n-1) a_{n-2}^{4},
\end{aligned}
$$

where again we fix $a_{0}^{3,4}=1$. The asymptotics of a general solution of Eq. (25) is a linear combination of $\psi_{3}$ and $\psi_{4}$.

It is well known [14] that the asymptotics, defined by the Thomè series, is not reached uniformly in $\arg (x)$. In our case, when the irregular singular point $x=\infty$ has the rank $R=3$, there exist $R+1=4$ Stokes rays $\arg (x)=\pi k / 4(k=$ $0,1,2,3)$, connecting singular points 0 and $\infty$ and dividing the complex plane $x$ in four sectors. In the general case, in any of these sectors and on any of the Stokes rays the asymptotics of the solution may be different. In the appendix it is shown that the asymptotics $\psi \sim$ const is reached in the area $-\frac{\pi}{2}<\arg (x)<\frac{\pi}{2}$, i.e. on the ray $[0,+\infty)$ and two adjacent sectors.

\section{Orthogonal scalar product}

One of the features of a non-Hermitian Hamiltonian is that its eigenstates $\psi_{\mathrm{N}}$, corresponding to different $E_{\mathrm{N}}$, are not orthogonal with respect to the usual scalar product, even if $\psi_{\mathrm{N}}$ form the complete basis. If we introduce, following [9], the operator $P$, changing the sign of $z$,

$P z P^{-1}=-z, \quad P \frac{d}{d z} P^{-1}=-\frac{d}{d z}$,

then it is easily seen that

$H^{+}=H[-\lambda]=P H P^{-1}$.

The operator $P$ is unitary and Hermitian in the original Fock space, since $\left\langle P \psi_{1} \mid P \psi_{2}\right\rangle \equiv\left\langle\psi_{1} \mid \psi_{2}\right\rangle$ and $\left\langle\psi_{1} \mid P \psi_{2}\right\rangle \equiv$ $\left\langle P \psi_{1} \mid \psi_{2}\right\rangle$ for the Bargmann scalar product. One can define the biorthogonal eigenvectors

$\bar{\psi}_{\mathrm{N}}=-P \psi_{\mathrm{N}}=-\psi_{\mathrm{N}}(-z)$

(the minus sign is convenient for the formulas in Sect. 6). If $H \psi_{\mathrm{N}}=E_{\mathrm{N}} \psi_{\mathrm{N}}$ with real $E_{\mathrm{N}}$, then

$H^{+} \bar{\psi}_{\mathrm{M}}=-H^{+} P \psi_{\mathrm{M}}=-P H \psi_{\mathrm{M}}=E_{\mathrm{N}} \bar{\psi}_{\mathrm{M}}$.

Using this and the adjoint equation $\left\langle\bar{\psi}_{\mathrm{M}}\right| H=E_{\mathrm{M}}\left\langle\bar{\psi}_{\mathrm{M}}\right|$, one obtains

$\left(E_{\mathrm{N}}-E_{\mathrm{M}}\right)\left\langle\bar{\psi}_{\mathrm{M}} \mid \psi_{\mathrm{N}}\right\rangle=\left\langle\bar{\psi}_{\mathrm{M}}|(H-H)| \psi_{\mathrm{N}}\right\rangle=0$,

so that $\left\langle\bar{\psi}_{\mathrm{M}} \mid \psi_{\mathrm{N}}\right\rangle=0$ when $E_{\mathrm{N}} \neq E_{\mathrm{M}}$. 
The scalar product

$$
\begin{aligned}
\left(\psi_{1} \mid \psi_{2}\right) & =-\left\langle\psi_{1}|P| \psi_{2}\right\rangle \equiv-\left\langle\psi_{1}(-z) \mid \psi_{2}(z)\right\rangle \\
& =-\int \frac{\mathrm{d} z \mathrm{~d} z^{*}}{2 \pi i} \mathrm{e}^{-z z^{*}} \psi_{1}^{*}(-z) \psi_{2}(z)
\end{aligned}
$$

has an orthogonality property but is, in the general case, not positively defined. Evidently, defining the Hermitian operator of the sign $v$ on the basis states

$$
\begin{aligned}
& \nu \psi_{\mathrm{N}}=v_{\mathrm{N}} \psi_{\mathrm{N}}, \quad \text { where } \\
& \begin{cases}v_{\mathrm{N}}=+1, & \text { if }\left\langle\psi_{\mathrm{N}}|P| \psi_{\mathrm{N}}\right\rangle>0, \\
v_{\mathrm{N}}=-1, & \text { if }\left\langle\psi_{\mathrm{N}}|P| \psi_{\mathrm{N}}\right\rangle<0,\end{cases}
\end{aligned}
$$

it is possible to introduce $\bar{\psi}_{\mathrm{N}}=v P \psi_{\mathrm{N}}$ and the positively defined scalar product (as in [9]) $\left(\psi_{1} \mid \psi_{2}\right)=\left\langle\psi_{1}|\nu P| \psi_{2}\right\rangle$, with respect to which the eigenstates are orthogonal. Note that $\left\langle\psi_{\mathrm{N}}|P| \psi_{\mathrm{N}}\right\rangle=0$ is impossible because it contradicts the completeness of the set of $\psi_{\mathrm{N}}$. The resolution of the identity then takes the form $[16,17]$

$$
I=\sum_{\mathrm{N}} \frac{\left|\psi_{\mathrm{N}}\right\rangle\left\langle\bar{\psi}_{\mathrm{N}}\right|}{\left\langle\bar{\psi}_{\mathrm{N}} \mid \psi_{\mathrm{N}}\right\rangle}
$$

However, this relation does not change if $\psi_{\mathrm{N}}$ and $\bar{\psi}_{\mathrm{N}}$ are multiplied on an arbitrary constant, so it is not necessary to choose the scalar product positively defined. For the sake of simplicity, the definition (34) will always be used.

The orthogonal scalar product may be defined in different ways $[16,17]$. For example, the standard scalar product of $L_{2}([0,+\infty))$ with the help of the transformation (20) induces the following scalar product:

$$
\begin{aligned}
\left(\psi_{1} \mid \psi_{2}\right) & =\left\langle\varphi_{1} \mid \varphi_{2}\right\rangle \\
& =\int_{0}^{+\infty} \mathrm{d} \xi \xi^{-1} \mathrm{e}^{-\frac{1}{2}\left(\xi^{2}-\frac{\mu}{\lambda}\right)^{2}} \psi_{1}^{*}\left(z=-i \xi^{2}\right) \psi_{2}\left(z=-i \xi^{2}\right) .
\end{aligned}
$$

Since Hamiltonian $H_{\xi}$ is Hermitian, its eigenstates $\varphi_{\mathrm{N}}$ with different $E_{\mathrm{N}}$ are orthogonal, so the states $\psi_{\mathrm{N}}$, connected to them by (20), are orthogonal with respect to (40). The inconvenience of this definition is that with respect to it the basis states $|n\rangle$ are not orthogonal, which makes the norm calculation for the wave functions, written in the form (27), very cumbersome.

\section{Numerical calculation of eigenvalues: the method and results}

Any solution (27) of Eq. (25) behaves at infinity as

$$
\psi(x) \underset{x \rightarrow \infty}{\sim} T_{3} \psi_{3}(x)+T_{4} \psi_{4}(x) .
$$

Here the constants $T_{3}$ and $T_{4}$, called connection factors, are functions of the two parameters $\beta$ and $\delta$ of (25). These constants $T_{3,4}$ do not depend on $x$ but can differ in different sectors of the complex plane, which are divided by Stokes rays. The expression for $T_{4}$ obtained in the appendix is valid on the half-plane $-\frac{\pi}{2}<\arg (x)<\frac{\pi}{2}$.

It follows from the consideration of Sect. 2 that $T_{4}=0$ is the quantization condition. For $\mu$ and $\lambda$ fixed and so for fixed $\beta=-\sqrt{2} \mu / \lambda$ the equation $T_{4}=0$ has to be solved for the variable $\delta=-2 \sqrt{2} E / \lambda$. From Eq. (25) and the definitions (26) it is evident that the ratio $E / \lambda$ can depend only on the ratio $\mu / \lambda$. Then all eigenvalues $E_{\mathrm{N}}$ are expressed in the form

$E_{\mathrm{N}}=-\lambda \frac{\delta_{\mathrm{N}}(\mu / \lambda)}{2 \sqrt{2}}$

where $\delta_{\mathrm{N}}$ are all solutions of the equation $T_{4}=0$.

In principle, all connection factors can be found using the general method for differential equations of the Heun class described in [14]. However, this method seems to be very complicated. For this reason we use a comparatively new and more simple method, proposed by the authors of [15] for the biconfluent Heun equation and based on the asymptotic series for Wronskians. It allows one to obtain the value of $T_{4}$ in the form of a transcendental expression,

$T_{4}=-\frac{1}{2}\left(\frac{\Gamma\left(\mathcal{N}+\frac{3}{2}\right)}{\left(\frac{1}{2}\right)^{\mathcal{N}+\frac{1}{2}}} g_{2 \mathcal{N}}+\frac{\Gamma(\mathcal{N}+2)}{\left(\frac{1}{2}\right)^{\mathcal{N}+1}} g_{2 \mathcal{N}+1}\right)$,

where $\mathcal{N}$ is an arbitrary positive integer and (see (63))

$g_{s}=\sum_{n=0}^{\infty} a_{n}^{3}\left(-\hat{c}_{s+n-1}-\beta \hat{c}_{s+n}-(s+2 n+2) \hat{c}_{s+n+1}\right)$.

The details of the method are described in the appendix, the definition of $\hat{c}_{n}$ is also given there, $a_{n}^{3}$ is defined in (31). The left side of the equation $T_{4}=0$ contains the infinite series in (44) with terms expressed via coefficients which are defined by recurrence relations. It is not clear if one can sum the series analytically, so we have used (43) for the numerical calculation of $\delta$ for the chosen set of ratios $\mu / \lambda$.

The numerical solutions of the equation $T_{4}=0$ were found $^{1}$ for all integer values of $\mu / \lambda$ from -11 to +11 . These values were chosen to compare our solutions with the results of [11]. To check the smooth dependence of the solutions on $\mu / \lambda$ in the vicinity of the peculiar value $\mu=0$ we also found the solutions for $\mu / \lambda= \pm 0.1, \pm 0.25, \pm 0.5$.

For the numerical calculation one can take a finite number $\mathcal{T}$ of terms in (44); thus $T_{4}$ becomes a polynomial in $\delta$. The convergence of solutions was checked by comparing the roots found for $\mathcal{T}-50$ and $\mathcal{T}$ terms in the equation. It is worth to note that to find roots with large absolute values one has to increase simultaneously the number of terms $\mathcal{T}$ and the precision of the calculations. We chose the limit of

\footnotetext{
1 All numerical calculations and graphical plotting were carried out using Maple computer algebra system.
} 


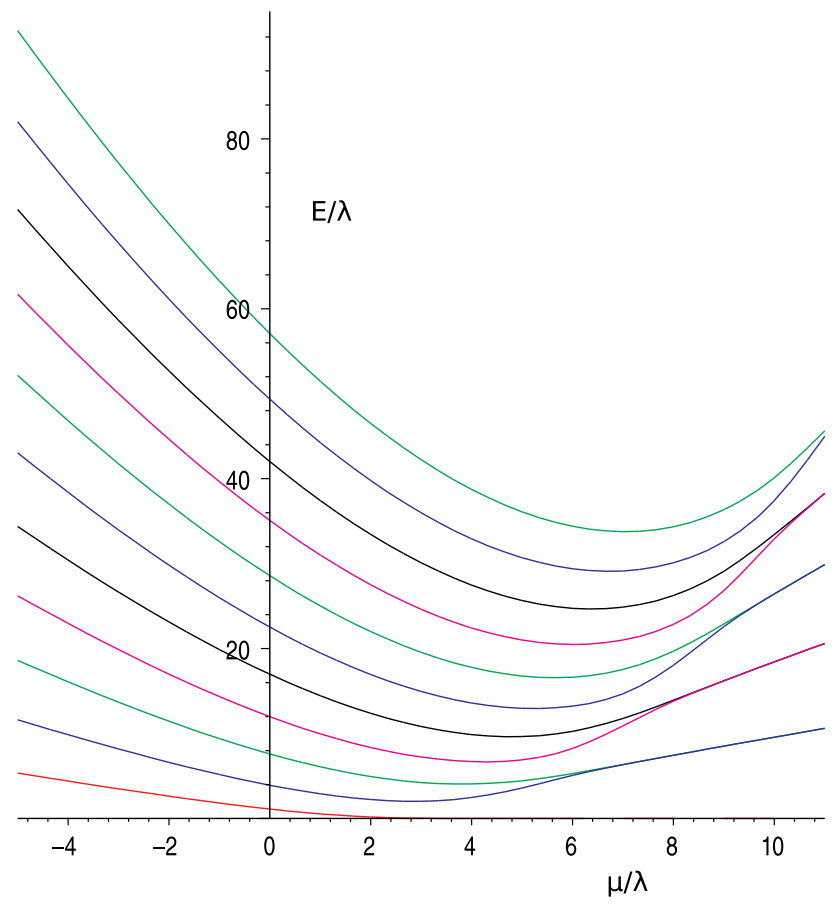

Fig. 1 Plot of the universal ratio $E / \lambda$ for the first 11 eigenvalues as functions of $\mu / \lambda$ from -5 to +11

absolute values of roots corresponding to $|E / \lambda|<99$. For this purpose it is sufficient to take $\mathcal{T}=400$ and to use the precision of 55 digits in the calculation. The independence of the roots on the arbitrary number $\mathcal{N}$ was not checked; we always set $\mathcal{N}=50$. However, we checked that for all considered $\mu / \lambda$ the equation has no real positive roots $\delta$ of the same order corresponding to negative energies. Positive roots with much larger values and also complex roots may appear as an artifact of the finiteness of $\mathcal{T}$.

The plots of the universal ratios $E_{\mathrm{N}} / \lambda$ for the first 11 eigenvalues (interpolated by cubic splines) are shown in Fig. 1 for $\mu / \lambda$ from -5 to +11 ; the plots in Fig. 2 are for the first five eigenvalues for $\mu / \lambda$ from -11 to +11 . These plots demonstrate the approximate double degeneracy of eigenvalues at $\mu / \lambda \rightarrow+\infty$ noted in [9]. In the data files applied to this article one can find non-trivial $(E \neq 0)$ ratios $E / \lambda$ with 20-digit precision.

The method of [15] was proposed recently and its applicability has not sufficiently been supported. Therefore we checked our eigenvalues by the usual "shooting" method. This test shows full agreement of the results obtained by both methods on the level of the adopted accuracy. We plan to devote a separate publication to the comparative analysis of different calculational algorithms for this eigenvalue problem.

With the known value of $\delta$ corresponding to the eigenvalue $E_{\mathrm{N}}$ one can find all coefficients of the series (27) for the eigenfunction from the recurrence relation (28). Since the values $\beta$ and $\delta$ determine the analytical eigenfunction

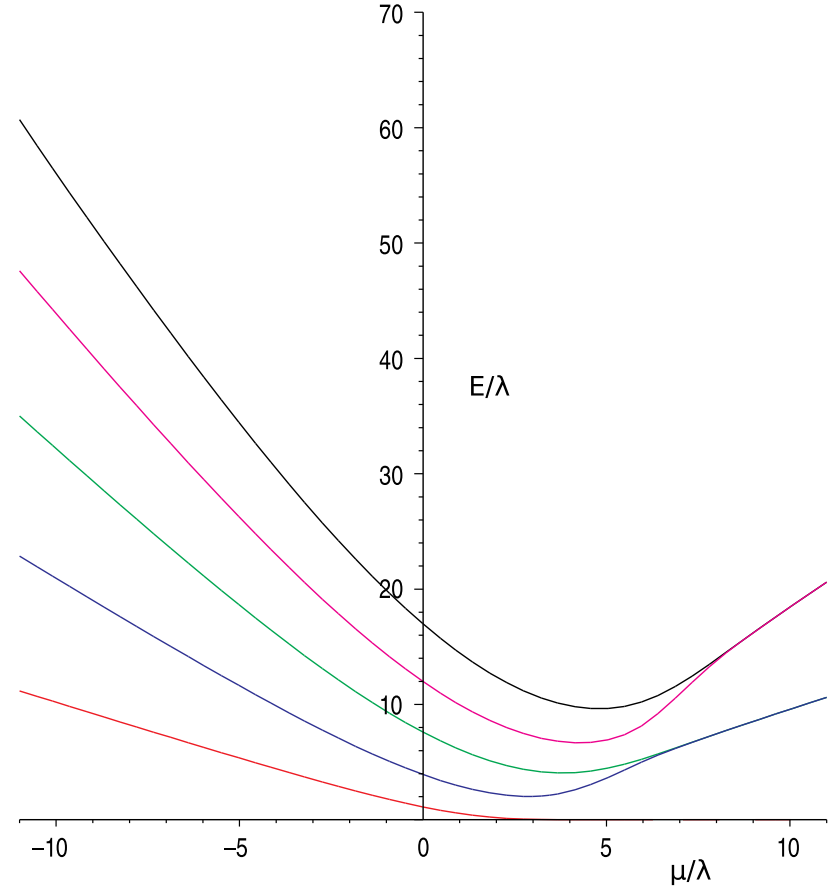

Fig. 2 Plot of the universal ratio $E / \lambda$ for the first five eigenvalues as functions of $\mu / \lambda$ from -11 to +11

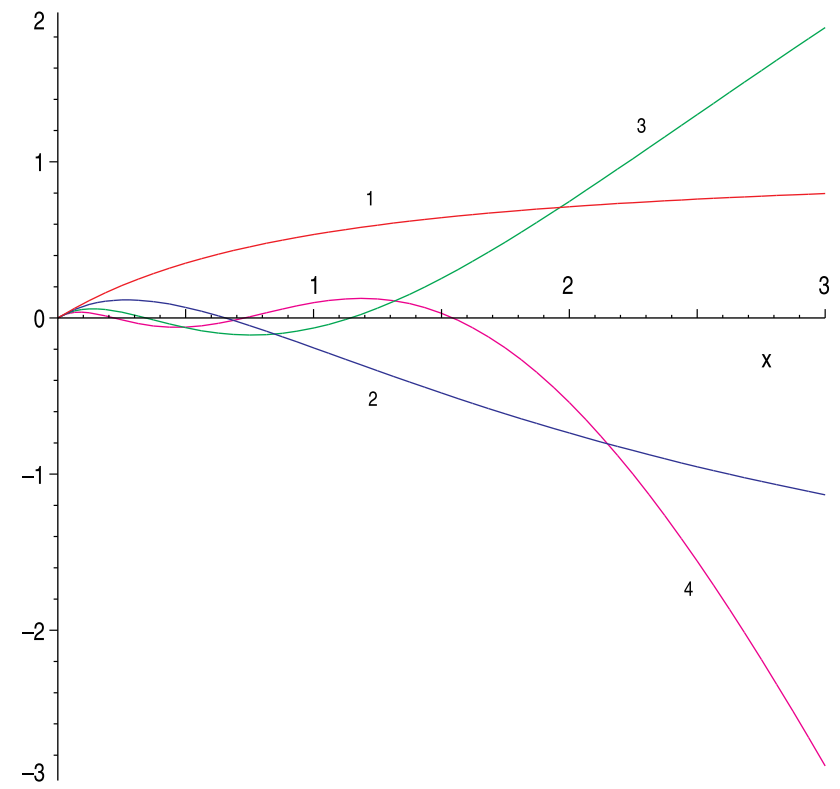

Fig. 3 Plot of the first four eigenfunctions for the case $\mu=0.1, \lambda=1$ at small $x$

uniquely, any eigenvalue $E_{\mathrm{N}}$ cannot be exactly degenerate. For example, in Fig. 3 plots of the first four eigenfunctions are shown for the "almost physical" case $\mu=0.1, \lambda=1$. Note that for small values $x<3$ the asymptotic behavior $\psi \sim$ const is not reached yet. These eigenfunctions have the usual properties- the $N$ th eigenfunction has $N$ zeros on the real positive axis including the point $x=0$. Obviously, 


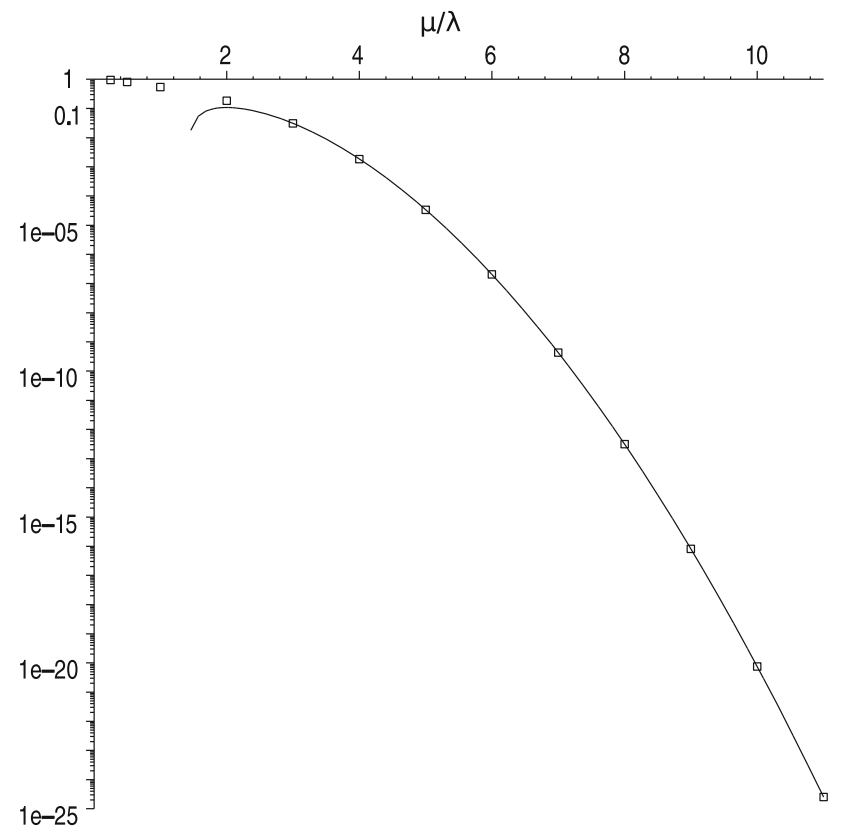

Fig. 4 Logarithmic plot of the ratio $E_{1} / \lambda$ for the first eigenvalue $N=1$ as a function of positive $\mu / \lambda$. The solid line shows theoretical value, small squares mark computed numerical values

the trivial vacuum eigenfunction $\psi_{0}=1$ with $N=0$ has no zeros.

One can compare the calculated numerical values of the first eigenvalue $N=1$ with the theoretical approximate value obtained in [9],

$\frac{E_{1}}{\lambda}=\frac{\mu^{2}}{\sqrt{2 \pi} \lambda^{2}} \mathrm{e}^{-\frac{\mu^{2}}{2 \lambda^{2}}}\left(1-\frac{2 \lambda^{2}}{\mu^{2}}+O\left(\frac{\lambda^{4}}{\mu^{4}}\right)\right)$.

In fact, this expression was found in a perturbative way: as the average value of the full Hamiltonian for the approximate eigenfunction, the parabolic cylinder function. This theoretical expression is valid for large positive values of $\mu / \lambda$, but it is not known how large the value has to be. The comparison shows that for $\mu / \lambda=5$ the relative error is less than 0.01 and it decreases at larger $\mu / \lambda$. In Fig. 4 the theoretical value (solid line) and the found numerical values (square points) for positive $\mu / \lambda$ are shown in a logarithmic scale.

\section{An application: calculation of the propagator}

As an application of the eigenvalues of the model (4) we consider the calculation of the one-particle state (one-pomeron state) propagator. By definition, this propagator is

$P(y)=\left\langle 1\left|\mathrm{e}^{-H y}\right| 1\right\rangle$.

The propagator as a function of the rapidity $y$ was found numerically in [11] by means of solving of the evolution equation in $y$ for the one-particle state. In [11] six cases were studied:
(1) $\mu=1, \lambda=0.1$;
(2) $\mu=1, \lambda=1 / 3$;
(3) $\mu=1, \lambda=1$;
(4) $\mu=0.1, \lambda=1$;
(5) $\mu=0, \lambda=1$;
(6) $\mu=-1, \lambda=0.1$.

To compare the results we calculated the propagator for the same values of the parameters $\mu$ and $\lambda$.

Inserting the expansion (39) between the initial and the final states in (46), one obtains the spectral representation

$$
\begin{aligned}
P(y) & =\sum_{\mathrm{N}} \frac{\left\langle 1\left|\mathrm{e}^{-H y}\right| \psi_{\mathrm{N}}\right\rangle\left\langle\bar{\psi}_{\mathrm{N}} \mid 1\right\rangle}{\left\langle\bar{\psi}_{\mathrm{N}} \mid \psi_{\mathrm{N}}\right\rangle} \\
& =\sum_{\mathrm{N}} \frac{\left\langle 1 \mid \psi_{\mathrm{N}}\right\rangle \mathrm{e}^{-E_{\mathrm{N}} y}\left\langle\bar{\psi}_{\mathrm{N}} \mid 1\right\rangle}{\left\langle\bar{\psi}_{\mathrm{N}} \mid \psi_{\mathrm{N}}\right\rangle} .
\end{aligned}
$$

The eigenfunction as a solution of the differential equation can be written as

$\psi_{\mathrm{N}}(z)=\sum_{n=0}^{\infty} c_{n}^{[N]} x^{n+1}=\sum_{n=0}^{\infty} c_{n}^{[N]}\left(\frac{i z}{\sqrt{2}}\right)^{n+1}$

with coefficients $c_{n}^{[N]}$ defined by the recurrence relation (28) using the found real eigenvalue. Thus all these coefficients are real and normalized by $c_{0}^{[N]}=1$. From this $\left\langle 1 \mid \psi_{\mathrm{N}}\right\rangle=$ $i / \sqrt{2}$ is obvious. Taking $\left\langle\bar{\psi}_{\mathrm{N}} \mid 1\right\rangle=-\left\langle 1 \mid \psi_{\mathrm{N}}(-z)\right\rangle^{*}=$ $\left\langle 1 \mid \psi_{\mathrm{N}}(z)\right\rangle^{*}=-i / \sqrt{2}$, one obtains

$P(y)=\frac{1}{2} \sum_{\mathrm{N}} \frac{\mathrm{e}^{-E_{\mathrm{N}} y}}{\left\langle\bar{\psi}_{\mathrm{N}} \mid \psi_{\mathrm{N}}\right\rangle}$.

The propagator has to satisfy the condition $P(0)=1$, which is a consequence of the completeness of the basis $\psi_{\mathrm{N}}$. A straightforward calculation gives

$\left\langle\bar{\psi}_{\mathrm{N}} \mid \psi_{\mathrm{N}}\right\rangle=\sum_{n=0}^{\infty} \frac{(-1)^{n}(n+1) !}{2^{n+1}}\left|c_{n}^{[N]}\right|^{2}$.

It is not a norm since it is not positively defined, but further we refer it to as "norm" for brevity. For $\mu<0$, when the finiteness condition for the Bargmann norm of eigenfunctions,

$\left\langle\psi_{\mathrm{N}} \mid \psi_{\mathrm{N}}\right\rangle=\sum_{n=0}^{\infty} \frac{(n+1) !}{2^{n+1}}\left|c_{n}^{[N]}\right|^{2}$,

can be imposed, the series (50) converges absolutely. For $\mu \geq 0$ the sum (50) is also finite, although (51) is not [9]. Our numerical calculations confirm this.

There are two sources of inaccuracy in this calculation. First, we know the eigenvalue with a finite precision, hence 
the equation $T_{4}=0$ is fulfilled only approximately. In correspondence with (41), an eigenfunction determined using an approximate eigenvalue has a contribution of the growing function $\psi_{4}$ with a very small coefficient. This contribution grows very rapidly, so at sufficiently large $|z|$ the approximate eigenfunction differs significantly from the exact one. In calculation it manifests itself in solving of the recurrence relations for $c_{n}^{[N]}$, where the cumulation of errors occurs which leads to bad convergence of (50). Second, one can take only a finite number of terms in the sum (50). Our calculation shows that the values of the "norms" depend on the number of terms (we got 1000, 2000, 5000, 10,000).

Since (48) coincides with the exact eigenfunction for small $|z|$ only, one can regularize the series (50) by introducing a cut-off in $|z|$ into the Bargmann scalar product (7). The factor $(n+1)$ ! in the numerator of $(50)$ is the Bargmann norm of $|n+1\rangle$. The introduction of the cut-off $|z|<L$ into the norm (8) leads to the substitution of $(n+1)$ ! with the integral

$\int_{0}^{L^{2}} \mathrm{~d} t \mathrm{e}^{-t} t^{n+1}=\Gamma(n+2)-\Gamma\left(n+2, L^{2}\right)$,

where $\Gamma(n, z)$ is the upper incomplete gamma function. The value $\mathrm{e}^{-L^{2}} L^{2(n+1)}$ is a measure of the inaccuracy for this regularization, hence one has to choose $L$ as large as possible. Also it is necessary to take into account the cut-off in the numerator of (47), which changes the factor $1 / 2$ in (49) to $B^{2} / 2$, where

$B=\Gamma(2)-\Gamma\left(2, L^{2}\right)=1-\mathrm{e}^{-L^{2}}\left(1+L^{2}\right)$.

Note that in all cases considered below the effect of the last substitution is negligible (the difference is less than $10^{-10}$ ).

By this means, we computed the "norms" $\left\langle\bar{\psi}_{\mathrm{N}} \mid \psi_{\mathrm{N}}\right\rangle$ for the first $N=1 \ldots 10$ eigenfunctions in the aforementioned cases (2)-(5) and for the first $N=1 \ldots 6$ eigenfunctions in case (6), taking 10,000 terms in (50). The regularization used effectively suppresses the contribution of higher powers $n$, so the sums of 5000 and 10,000 terms practically coincide. We checked the independence of "norms" on the cut-off, varying $L^{2}$ within wide limits. We have begun with small $L^{2}=10$ and sequentially enlarged $L^{2}$, checking that the "norm" does not change with a relative precision of at least 0.001 . Starting with some value of $L^{2}$ the regularized "norms" became anomalously large. Then we restored the previous value of $L^{2}$ and used it for calculating of "norms" in propagator (49). Here are the values of the cut-off $L^{2}$ for which the "norms" do not change in the cases considered:

(2) $-L^{2}$ from 25 to $100,(3)-L^{2}$ from 25 to 1200 ,

(4) and (5) $-L^{2}$ from 25 to 4000, (6) $-L^{2}$ from 10 to 30 .

The condition $P(0)=1$, which is a consequence of the completeness condition, is fulfilled with an error of 0.0025 in case (2) and less than $10^{-5}$ in cases (3)-(6). One can see that the larger the value of $\mu / \lambda$, the smaller the interval of $|z|$ for which the approximated eigenfunctions are in good coincidence with the exact ones.

The calculation shows that not all "norms" are positive. In the considered cases their signs alternate for sequential $N$. Perhaps, it can be explained by the behavior of the eigenfunctions (see Fig. 3) on the real axis $x$. For all solutions $\psi^{\prime}(0)=$ 1 is implied. The function $\psi_{\mathrm{N}}(x)$ has $N$ zeros, including $x=0$, and its asymptotics at large $x>0$ is constant, so the sign of this constant is $(-1)^{N-1}$. At $x \rightarrow-\infty$ the function grows most rapidly, as $\psi_{\mathrm{N}}(x) \sim \exp \left(x^{2}+\beta x\right) / x$, and it has a negative sign. Hence, the main contribution to the "norm" (37) comes from the real axis $x$ and the sign of this contribution, if the common "-" is taken into account, coincides with the sign of the constant.

However, case (1) with large positive $\mu / \lambda$ differs radically from the others. Here it is impossible to select an interval of $L^{2}$ for which all values of "norms" $\left\langle\bar{\psi}_{\mathrm{N}} \mid \psi_{\mathrm{N}}\right\rangle$ do not depend on the cut-off. We considered the condition $P(0)=1$ and the alternation of signs of "norms" as a criterion of success. We tried many variants from $L^{2}=0.5$ to $L^{2}=100$, but we cannot find any acceptable result. For case (1) we also tried to use the resolution of the identity, analogous to (39) but induced by the scalar product (40) with the cut-off $\xi<$ $L$ for the integration over $\xi$. We could reliably find only the first coefficient $1 /\left(\psi_{1} \mid \psi_{1}\right)$ of the expansion, the others significantly depend on the cut-off.

Thus our approach to the calculation of propagator, based on the power series expansion of eigenfunctions, does not allow us to find a reliable answer in the case of large positive $\mu / \lambda$. It is worth to note that just in the case of large positive $\mu / \lambda$ the analytical methods of [9] work well.

In Fig. 5 plots of the propagator as a function of the rapidity $y$ are shown in a logarithmic scale. The numeration of curves $2-5$ coincides with that of the considered cases. In the calculation of (49) 10 eigenfunctions for the cases (2)-(5) and six eigenfunctions for case (6) were taken into account. The general appearance of the curves in Fig. 5 is in full agreement with the curves in Fig. 1 from [11]. The comparison of the values of the propagators with the original numerical data for Fig. 1 from [11] at integer $y$ from 0 to 20 (plots are shown only for $y<10$ ) demonstrates that they coincide with a relative precision of less than 0.002 for all $y>0$.

In case (2) the propagator grows at small $y$. The physical propagator tends to zero at $y \rightarrow+\infty$, so this growth has to eventually change to a decrease. Theoretically, since the propagator is defined by (49) with positive values $E_{\mathrm{N}}$ (which are growing with $N$ ), at sufficiently large $y$ only the decreasing contribution of $N=1$ remains. In Fig. 6 the plot of $P(y)$ for $y$ from 1 to 1000 in case (2) is shown with both axes given in a logarithmic scale. It confirms the decrease of the propagator at $y>10$. 


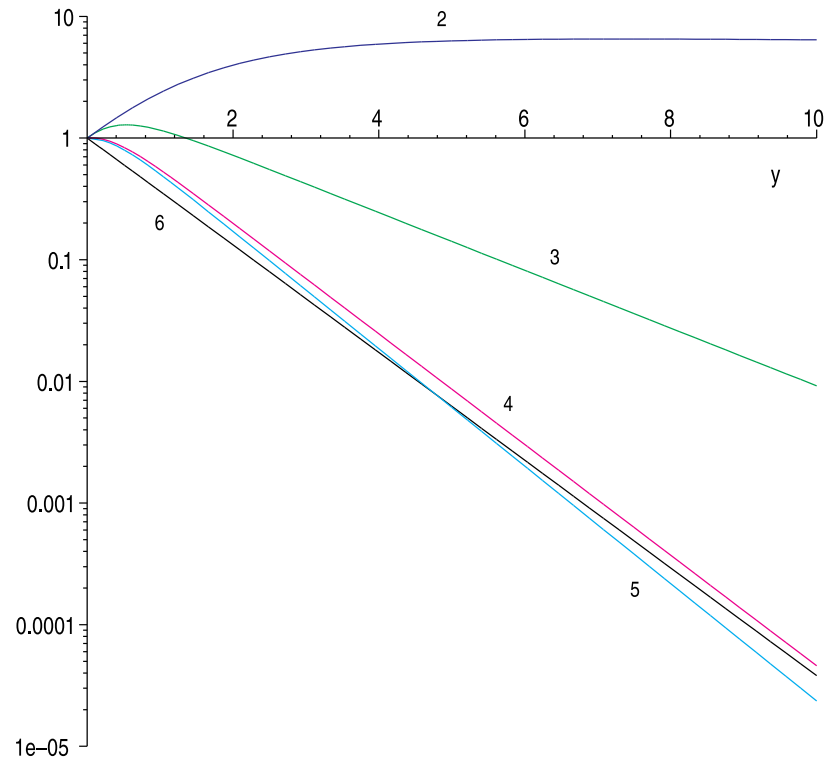

Fig. 5 Full propagators for different values $\mu$ and $\lambda$ as functions of $y$. (2) $\mu=1, \lambda=1 / 3$; (3) $\mu=1, \lambda=1$; (4) $\mu=0.1, \lambda=1$; (5) $\mu=0$, $\lambda=1$; (6) $\mu=-1, \lambda=0.1$

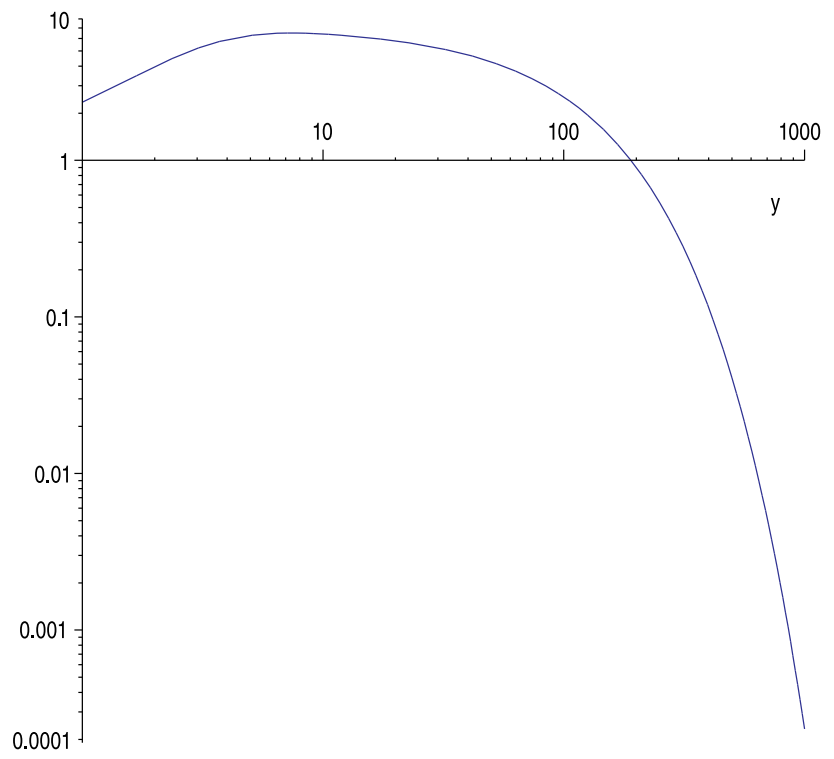

Fig. 6 Full propagator for $\mu=1$ and $\lambda=1 / 3$ as a function of $y$ with both axes given in the logarithmic scale

\section{Conclusions}

In the present work the one-dimensional reggeon model was considered in its equivalent form of the quantum mechanics in imaginary time. Since the Hamiltonian of the model is non-Hermitian, an indefinite scalar product with respect to which the eigenfunctions are orthogonal had to be introduced. This allows one to write the correct resolution of the identity and, hence, the spectral representation for the propagator. The similarity transformation is known to turn the
Hamiltonian into a Hermitian form [7], which establishes the completeness of the basis of eigenfunctions and the reality of eigenvalues.

The choice of the quantization conditions in this model is not trivial [9]. The condition of finiteness of the norm in the Fock-Bargmann space can be imposed only for negative values of parameter $\mu$, i.e. in the area of applicability of the perturbation theory. For $\mu \geq 0$ the asymptotical conditions for the eigenfunctions have to be chosen in the same manner as in the case $\mu<0$; then the energies are real and positive. In the case $\mu \geq 0$ the asymptotical conditions do not lead to the finite Bargmann norm, but the non-positive "norm" connected with the indefinite scalar product is finite for all cases.

The eigenfunction equation has the canonical form of the biconfluent Heun equation for solutions of which the asymptotical conditions are implied. By resolving these conditions, using the new method of [15], we can derive the equation which completely defines the eigenvalues. This equation is transcendental, because it contains an infinite sum of polynomials, so that we can solve it only numerically. For the chosen sets of parameters of the model (in fact, the only parameter is the ratio $\mu / \lambda$ ) we found several eigenvalues of the energy.

We used the found values of energies for a calculation of the pomeron propagator. In principle, knowing the eigenvalues and eigenfunctions, one can apply the spectral representation. The problem is that we express eigenfunctions as power series and their coefficients are defined with cumulative errors, even if we know the eigenvalues with high precision. These errors lead to bad convergence of the series for the scalar products appearing as coefficients of the spectral representation. To provide convergence of the series we introduced a cut-off into the integration which defines the scalar product. This allows one to calculate the propagator, excluding the case of large positive $\mu / \lambda$, when the scalar products depend significantly on the cut-off. So this method gives satisfactory results only for values of $\mu / \lambda$ not very large, just when the perturbative theory does not work. In this case we find full agreement with previous straightforward numerical calculations [11].

Acknowledgements The authors are thankful to N.V. Antonov, M.V. Ioffe, M.V. Komarova, M.V. Kompaniets, V.N. Kovalenko for very useful discussions.

Data Availability Statement This manuscript has data included as electronic supplementary material. The online version of this article contains supplementary material, which is available to authorized users.

Open Access This article is distributed under the terms of the Creative Commons Attribution 4.0 International License (http://creativecomm ons.org/licenses/by/4.0/), which permits unrestricted use, distribution, and reproduction in any medium, provided you give appropriate credit to the original author(s) and the source, provide a link to the Creative Commons license, and indicate if changes were made.

Funded by SCOAP ${ }^{3}$. 


\section{Appendix: Calculation of the connection factor $T_{4}$}

The following method of calculation of the connection factors was presented in [15]. Let us make a transition from the function $\psi(x)$ following (23) with $\alpha=-1$ to $u(x)$, which is a solution of the Heun equation in the normal form (24). Accordingly, for the asymptotics at $x \rightarrow \infty$ one can write

$u(x) \underset{x \rightarrow \infty}{\sim} T_{3} u_{3}(x)+T_{4} u_{4}(x)$,

where $u_{3,4}(x)=\mathrm{e}^{-\frac{\beta x}{2}-\frac{x^{2}}{2}} \psi_{3,4}(x)$. Let us denote a Wronskian of two functions by $\mathcal{W}[f, g]=f g^{\prime}-f^{\prime} g$. Forming the Wronskian of both sides of (54) with $u_{3}(x)$ one obtains

$\mathcal{W}\left[u, u_{3}\right]=T_{4} \mathcal{W}\left[u_{4}, u_{3}\right]$.

Since $u, u_{3}$ and $u_{4}$ are solutions of Eq. (24), expressing their second derivatives from this equation one obtains $\mathcal{W}^{\prime}\left[u, u_{3}\right]=0$ and $\mathcal{W}^{\prime}\left[u_{4}, u_{3}\right]=0$; hence these Wronskians are constants and do not depend on $x$. Then to calculate $\mathcal{W}\left[u_{4}, u_{3}\right]$ it is enough to use only first terms of asymptotical expansions (30) and after brief calculations one obtains $\mathcal{W}\left[u_{4}, u_{3}\right]=-\mathcal{W}\left[u_{3}, u_{4}\right]=-2$.

Introducing the auxiliary functions

$v(x)=\exp \left(\frac{x^{2}}{4}\right) u(x), \quad v_{3}(x)=\exp \left(\frac{x^{2}}{4}\right) u_{3}(x)$,

for their Wronskian one obtains

$\mathcal{W}\left[v(x), v_{3}(x)\right]=\mathcal{W}\left[u, u_{3}\right] \exp \left(\frac{x^{2}}{2}\right)$.

We address the constant $\mathcal{W}\left[u, u_{3}\right]$ by comparing asymptotical expansions of the left and right hand sides. Using (23) and (56) for the left hand side one obtains

$$
\begin{aligned}
\mathcal{W}\left[v(x), v_{3}(x)\right] & =\mathcal{W}\left[\mathrm{e}^{\frac{\beta x}{2}+\frac{x^{2}}{4}} \hat{u}, \mathrm{e}^{-\frac{\beta x}{2}-\frac{x^{2}}{4}} \psi_{3}\right] \\
& =-(x+\beta) \hat{u} \psi_{3}+\hat{u} \psi_{3}^{\prime}-\hat{u}^{\prime} \psi_{3},
\end{aligned}
$$

where

$\hat{u}(x)=\exp \left(-\frac{\beta x}{2}\right) u(x)$

The function $\hat{u}(x)$ is analytical, its power series is

$\hat{u}(x)=\sum_{n=0}^{\infty} \hat{c_{n}} x^{n+1}$

and it converges in the entire complex plane $x$. The coefficients are defined by the recurrence relation

$$
\begin{aligned}
& n(n+1) \hat{c}_{n}=\left(-\beta n+\frac{\delta}{2}\right) \hat{c}_{n-1}-\hat{c}_{n-2} \\
& \quad+\beta \hat{c}_{n-3}+\hat{c}_{n-4}, \quad \hat{c}_{0}=1 \quad\left(\hat{c}_{n}=0 \text { if } n<0\right) .
\end{aligned}
$$

Substituting into (58) the power series from (60) and the asymptotical expansion (30) for $\psi_{3}$ one can obtain the asymptotical power series

$\mathcal{W}\left[v(x), v_{3}(x)\right] \sim \sum_{s=-\infty}^{\infty} g_{s} x^{s+1}$,

where

$g_{s}=\sum_{n=0}^{\infty} a_{n}^{3}\left(-\hat{c}_{s+n-1}-\beta \hat{c}_{s+n}-(s+2 n+2) \hat{c}_{s+n+1}\right)$.

In order to obtain the right hand side asymptotical expansion in (57) one should use the Heaviside exponential series [18]

$\mathrm{e}^{t} \sim \sum_{n=-\infty}^{\infty} \frac{t^{n+\Delta}}{\Gamma(n+1+\Delta)}$

This asymptotical expansion is valid for arbitrary $\Delta \in \mathbb{C}$ and $|\arg (t)|<\pi$. If on the right hand side of (57) one expresses $\mathcal{W}\left[u, u_{3}\right]=\eta_{0}+\eta_{1}$, where $\eta_{0,1}$ are unknown constants, then

$$
\begin{aligned}
\mathcal{W} & {\left[u, u_{3}\right] \exp \left(\frac{x^{2}}{2}\right) } \\
= & \sum_{n=-\infty}^{\infty}\left(\eta_{0} \frac{\left(\frac{x^{2}}{2}\right)^{n+\frac{1}{2}}}{\Gamma\left(n+\frac{3}{2}\right)}+\eta_{1} \frac{\left(\frac{x^{2}}{2}\right)^{n+1}}{\Gamma(n+2)}\right),
\end{aligned}
$$

where in the first and second terms $\Delta=1 / 2$ and $\Delta=1$, respectively. Thus the sum in the right part of (65) contains all integer powers in $x$. By comparison of the coefficients of $x^{2 \mathcal{N}}$ and $x^{2 \mathcal{N}+1}(\mathcal{N}$ is an integer positive number) in (62) and (65) $\eta_{0}$ and $\eta_{1}$ can be defined and then one obtains

$\mathcal{W}\left[u, u_{3}\right]=\frac{\Gamma\left(\mathcal{N}+\frac{3}{2}\right)}{\left(\frac{1}{2}\right)^{\mathcal{N}+\frac{1}{2}}} g_{2 \mathcal{N}}+\frac{\Gamma(\mathcal{N}+2)}{\left(\frac{1}{2}\right)^{\mathcal{N}+1}} g_{2 \mathcal{N}+1}$.

By construction the right hand side does not depend on $\mathcal{N}$. The recurrence relations (31) and (61) define $a_{n}^{3}$ and $\hat{c}_{n}$ as polynomials in $\beta$ and $\delta$, but the expression for $g_{s}$ contains an infinite sum. Thus, in the general case, $\mathcal{W}\left[u, u_{3}\right]$ is a transcendent function of the parameters.

Substituting (66) and $\mathcal{W}\left[u_{4}, u_{3}\right]=-2$ into (55), one obtains (43). The Heaviside expansion in (65) can be used when $\left|\arg \left(x^{2} / 2\right)\right|<\pi$; therefore Eq. (43) for the connection factor is valid only when $-\pi / 2<\arg (x)<\pi / 2$.

\section{References}

1. V.N. Gribov, Sov. Phys. JETP 26, 414 (1968)

2. A.A. Migdal, A.M. Polyakov, K.A. Ter-Martirosyan, Phys. Lett. 48 B, 239 (1974) 
3. A.A. Migdal, A.M. Polyakov, K.A. Ter-Martirosyan, Sov. Phys. JETP 40, 420 (1975)

4. J.L. Cardy, R.L. Sugar, J. Phys. A: Math. Gen. 13, L423 (1980)

5. D. Amati, L. Caneschi, R. Jengo, Nucl. Phys. B 101, 397 (1975)

6. V. Alessandrini, D. Amati, R. Jengo, Nucl. Phys. B 108, 425 (1976)

7. R. Jengo, Nucl. Phys. B 108, 447 (1976)

8. D. Amati, M. Le Bellac, G. Marchesini, M. Ciafaloni, Nucl. Phys. B 112, 107 (1976)

9. M. Ciafaloni, M. Le Bellac, G.C. Rossi, Nucl. Phys. B 130, 388 (1977)

10. A. Schwimmer, Nucl. Phys. B 94, 445 (1975)
11. M.A. Braun, G.P. Vacca, Eur. Phys. J. C 50, 857 (2007)

12. M.A. Braun, Eur. Phys. J. C 77, 49 (2017)

13. S. Bondarenko, Eur. Phys. J. C 71, 1587 (2011)

14. S.Yu Slavyanov, W. Lay, Special functions: a unified theory based on singularities (Oxford University Press, London, 2000)

15. E.M. Ferreira, J. Sesma, Numer. Algorithms 71, 797 (2016)

16. A. Mostafazadeh, J. Math. Phys 43, 205 (2002)

17. A. Mostafazadeh, J. Math. Phys 43, 2814 (2002)

18. G.H. Hardy, Divergent series (Oxford University Press, London, 1956) 